(C) 1998 OPA (Overseas Publishers Association) Amsterdam B.V. Published under license under the Gordon and Breach Science Publishers imprint. Printed in India.

\title{
NOVEL CURRENT-CONVEYOR-BASED UNIVERSAL CURRENT-MODE BIQUAD FILTER WITH THREE INPUTS AND ONE OUTPUT
}

\author{
MUHAMMAD TAHER ABUELMA'ATTI* \\ and HUSSAIN ABDULLAH AL-ZAHER
}

King Fahd University of Petroleum and Minerals, Box 203, Dhahran 31261, Saudi Arabia

(Received 19 August 1997; In final form 3 November 1997)

\begin{abstract}
A novel universal current-mode filter with three inputs and one high imedance output is presented. The proposed circuit uses four plus-type second-generation currentconveyors, grounded resistors and grounded capacitors. The proposed circuit enjoys low active and passive sensitivities and independent control of the parameters $\omega_{o}$ and $\omega_{o} / Q_{o}$ using grounded resistors.

Keywords: Current conveyors; active filters
\end{abstract}

\section{INTRODUCTION}

Recently, Chang, Chien and Wang, 1994, proposed a universal active current filter with three inputs and one ouput using current conveyors. The proposed circuit uses two plus-type first-generation currentconveyors, two minus-type second-generation current conveyors, two grounded capacitors and two grounded resistors and enjoys the following attractive features:

1. Low filter sensitivity to passive components.

2. The use of grounded capacitors which is attractive for integrated circuit implementation.

\footnotetext{
${ }^{*}$ Corresponding author.
} 
3. The versatility to synthesize virtually any type of active filter transfer function.

However, the circuit suffers from the following disadvantages:

1. Use of different types of current conveyors.

2. Interdependent control of the parameters $\omega_{o}$ and $\omega_{o} / Q_{o}$. Thus, while the parameter $\omega_{o}$ can be adjusted without disturbing the parameter $\omega_{o} / Q_{o}$, the parameter $\omega_{o} / Q_{o}$ cannot be adjusted without disturbing the parameter $\omega_{o}$.

3. The sensitivity of the circuit to the voltage and current tracking errors of the current conveyors is not clear.

This paper presents a novel three-input universal current-mode biquad active filter. The proposed circuit enjoys the following attractive features:

1. Use of one type of second-generation current-conveyor.

2. Independent control of the parameters $\omega_{o}$ and $\omega_{o} / Q_{o}$. Thus the parameter $\omega_{o}$ can be adjusted without disturbing the parameter $\omega_{o} /$ $Q_{o}$, and the parameter $\omega_{o} / Q_{o}$, can be adjusted without disturbing the parameter $\omega_{o}$.

3. Enjoys low active and passive sensitivities.

4. Use of grounded capacitors and grounded resistors.

\section{PROPOSED CIRCUIT}

The proposed circuit is shown in Figure 1. The circuit uses plus-type second-generation current-conveyors $(\mathrm{CCII}+)$ only. Using the standard notation, the CCII + characteristics can be described by $i_{z}=\alpha i_{x}$, $\nu_{x}=\beta \nu_{y}$, where $\alpha=1-\varepsilon_{i}$ and $\varepsilon_{i}$ denotes the current-tracking error, $\beta=1-\varepsilon_{\nu}$ and $\varepsilon_{\nu}$ denotes the voltage-tracking error. The single output current $I_{o}$ can be expressed as

$$
I_{o}=\frac{\alpha_{4} \beta_{4} G_{7}}{G_{4}} \frac{s^{2} C_{1} C_{3} \alpha_{2} \beta_{2} I_{3}-s C_{1} G_{4} \alpha_{2} I_{2}+G_{2} G_{4} \alpha_{1} \alpha_{2} \alpha_{3} \beta_{1} I_{1}}{s^{2} C_{1} C_{6}+s C_{1} G_{6}+G_{2} G_{5} \alpha_{1} \alpha_{2} \alpha_{3} \beta_{1} \beta_{3}}
$$

From (1) the parameters $\omega_{o}$ and $\omega_{o} / Q_{o}$ can be expressed as

$$
\omega_{o}^{2}=\frac{\alpha_{1} \alpha_{2} \alpha_{3} \beta_{1} \beta_{3} G_{2} G_{5}}{C_{1} C_{6}}
$$




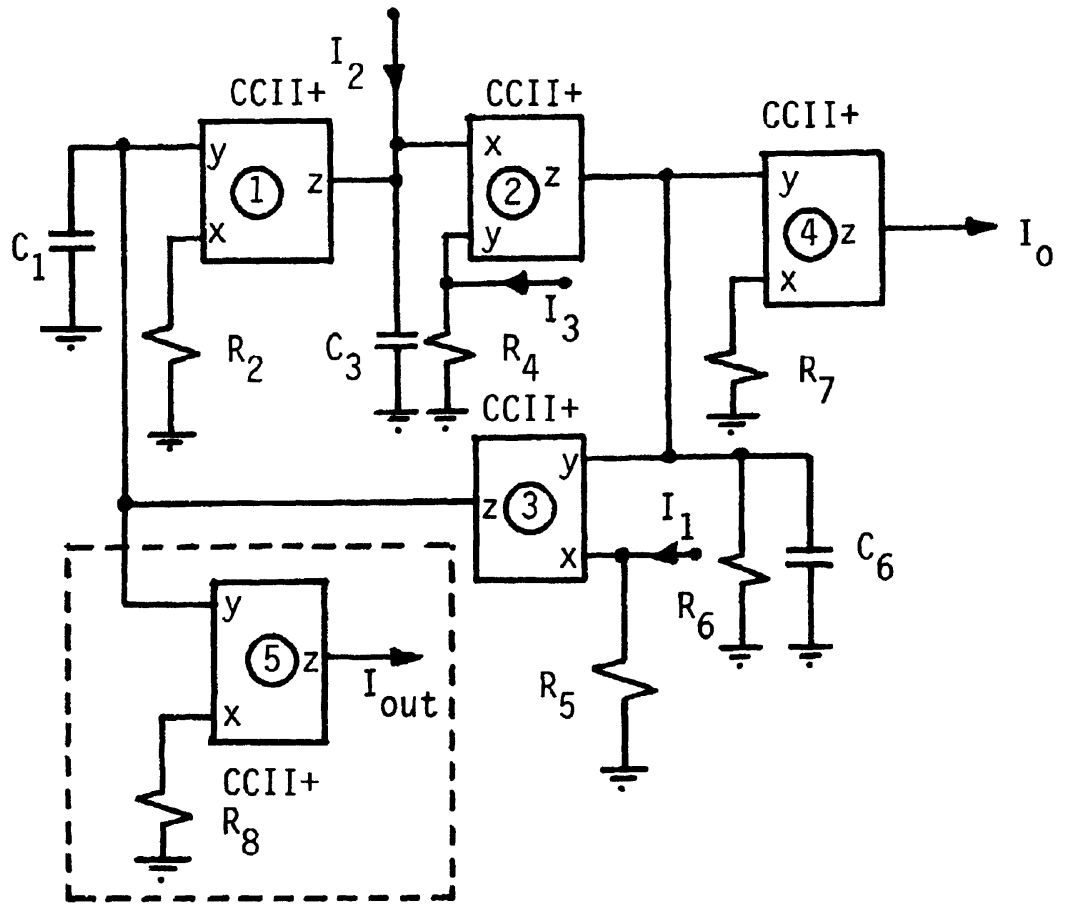

FIGURE 1 Proposed universal current-mode biquad Filter.

and

$$
\frac{\omega_{o}}{Q_{o}}=\frac{G_{6}}{C_{6}}
$$

From (1) it can be seen that:

1. The lowpass response can be realised with $I_{2}=I_{3}=0$

2. The highpass response can be obtained with $I_{1}=I_{2}=0$

3. The bandpass response can be obtained with $I_{1}=I_{3}=0$

4. The notch response can be obtained with $I_{2}=0$ and $I_{1}=I_{3}$

5. The allpass response can be obtained with $I_{1}=I_{2}=I_{3}, G_{4}=G_{5}$ and $C_{3}=C_{6}$.

From (1) it can also be seen that the lowpass gain, the highpass gain and the bandpass gain are approximately given by

$$
G_{\mathrm{LP}} \cong \frac{G_{7}}{G_{5}}
$$




$$
G_{\mathrm{HP}} \cong \frac{G_{7}}{G_{4}} \frac{C_{3}}{C_{6}}
$$

and

$$
G_{\mathrm{BP}} \cong \frac{G_{7}}{G_{6}}
$$

From (2) - (6) it can be seen that the parameter $\omega_{0}$ can be adjusted by controlling the resistance $R_{2}=1 / G_{2}$ without disturbing the parameters $\omega_{o} / Q_{o}, G_{\mathrm{LP}}, G_{\mathrm{HP}}$ and $G_{\mathrm{BP}}$. Also, the highpass gain can be adjusted by controlling the resistance $R_{4}=1 / G_{4}$ without disturbing the parameters $\omega_{o}, \omega_{o} / Q_{o}, G_{\mathrm{LP}}$ and $G_{\mathrm{BP}}$. Moreover, the parameter $\omega_{o} /$ $Q_{o}$ can be adjusted by controlling the resistance $R_{6}=1 / G_{6}$ without disturbing the parameter $\omega_{o}$. However, controlling the resistance $R_{6}$ will disturb the bandpass gain $G_{\mathrm{BP}}$. A possible strategy for adjusting the parameters $\omega_{o}, \omega_{o} / Q_{o}, G_{\mathrm{LP}}, G_{\mathrm{HP}}$ and $G_{\mathrm{BP}}$ is, therefore, as follows: First the resistance $R_{6}=1 / G_{6}$ is controlled to adjust the parameter $\omega_{o} /$ $Q_{o}$, then the resistor $R_{7}=1 / G_{7}$ is controlled to adjust the bandpass gain $G_{\mathrm{BP}}$; the resistance $R_{4}=1 / G_{4}$ is controlled to adjust the highpass gain $G_{\mathrm{HP}}$; the resistance $R_{5}=1 / G_{5}$ is controlled to adjust the lowpass gain $G_{\mathrm{LP}}$, and finally the resistance $R_{2}=1 / G_{2}$ is adjusted to control the parameter $\omega_{o}$.

From (2) and (3) it is easy to show that the active and passive sensitivities of the parameters $\omega_{o}$ and $Q_{o}$ are

$$
\begin{gathered}
S_{R_{2}}^{\omega_{o}}=S_{R_{5}}^{\omega_{o}}=S_{C_{1}}^{\omega_{o}}=S_{C_{6}}^{\omega_{o}}=-S_{\alpha_{1}}^{\omega_{o}}=-S_{\alpha_{2}}^{\omega_{o}}=-S_{\alpha_{3}}^{\omega_{o}} \\
=-S_{\beta_{1}}^{\omega_{o}}=-S_{\beta_{3}}^{\omega_{o}}=-\frac{1}{2} \\
S_{R_{2}}^{Q_{o}}=S_{R_{5}}^{Q_{o}}=S_{C_{1}}^{Q_{o}}=-S_{C_{6}}^{Q_{o}}=-S_{\alpha_{1}}^{Q_{o}}=-S_{\alpha_{2}}^{Q_{o}} \\
=-S_{\alpha_{3}}^{Q_{o}}=-S_{\beta_{1}}^{Q_{o}}=-S_{\beta_{3}}^{Q_{o}}=-\frac{1}{2} \\
S_{R_{6}}^{Q_{o}}=1, \quad S_{\alpha_{4}}^{\omega_{o}}=S_{\beta_{4}}^{\omega_{o}}=S_{\alpha_{4}}^{Q_{o}}=S_{\beta_{4}}^{Q_{o}}=S_{\beta_{2}}^{\omega_{o}}=S_{\beta_{2}}^{Q_{o}}=0 \\
S_{R_{4}}^{\omega_{o}}=S_{R_{4}}^{Q_{o}}=S_{R_{7}}^{Q_{o}}=S_{R_{7}}^{\omega_{o}}=0
\end{gathered}
$$

Thus, all the active and passive sensitivities are no more than unity. 
It is worth mentioning here that, another output current can be obtained when $I_{1}=0$. By using an additional second-generation current-conveyor and a grounded resistor as shown in the dotted box of Figure 1; this addition is, however, optional, the new output current can be expressed as

$$
I_{\mathrm{out}}=\alpha_{3} \alpha_{4} \beta_{3} \beta_{4} \frac{G_{8}}{G_{7}} \frac{G_{5}}{s C_{1}} I_{o}
$$

Thus, when $I_{o}$ is realising a bandpass response, the current $I_{\text {out }}$ will realise a lowpass response. Also, when $I_{o}$ is realising a highpass response, the current $I_{\text {out }}$ will realise a bandpass response.

\section{EXPERIMENTAL RESULTS}

To verify the theoretical analysis, the proposed circuit was used to realise LP, HP, BP and notch filters using the AD844 current-conveyor.

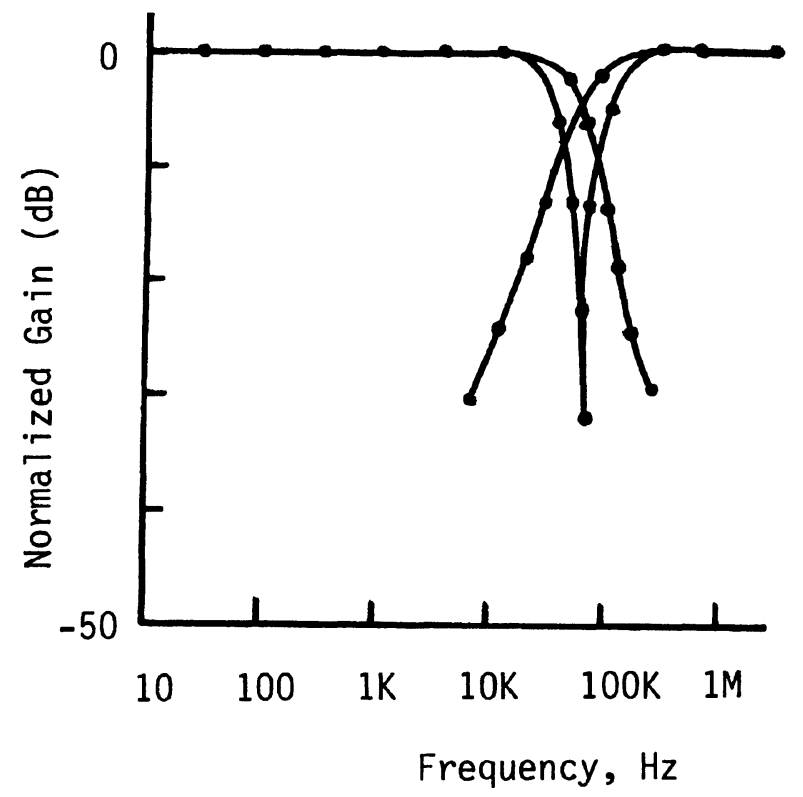

FIGURE 2 Measured lowpass, highpass and notch responses. $C_{1}=C_{3}=C_{6}=470 \mathrm{PF}$, $R_{2}=4 \mathrm{~K}, R_{4}=R_{5}=R_{6}=R_{7}=5 \mathrm{~K}$. 
The results obtained with $C_{1}=C_{3}=C_{6}=470 \mathrm{pF}, R_{2}=4 \mathrm{~K}, R_{4}=R_{5}=$ $R_{6}=R_{7}=5 \mathrm{~K}$ are shown in Figure 2 . These results are in good agreement with the theoretical analysis.

\section{CONCLUSION}

A new universal current-mode filter has been presented. The proposed filter offers the following advantages:

(i) Use only one type of current-conveyors (CCII +).

(ii) All resistors and capacitors are grounded.

(iii) Low active and passive sensitivities.

(iv) Independent control of the parameters $\omega_{o}$ and $\omega_{o} / Q_{o}$ using grounded resistors.

(v) High output impedance.

(vi) All the standard filter functions are realised with no component matching requirement except for all allpass realisation.

\section{References}

Chang, C.-M., Chien, C.-C. and Wang, H.-Y. (1994) Universal active current filter with three inputs using current conveyors-Part 2, International Journal of Electroncis, 76, 87-89. 

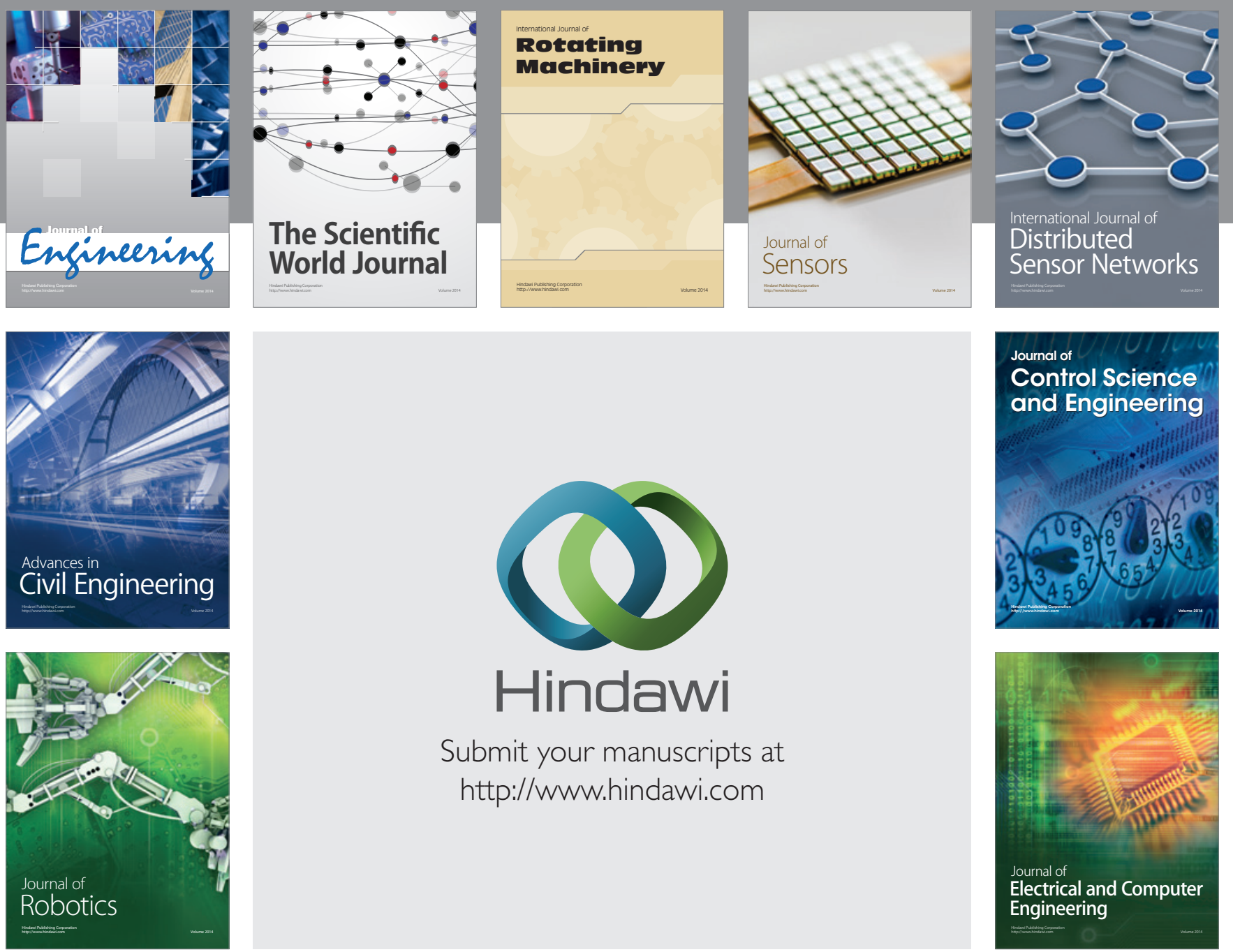

Submit your manuscripts at

http://www.hindawi.com
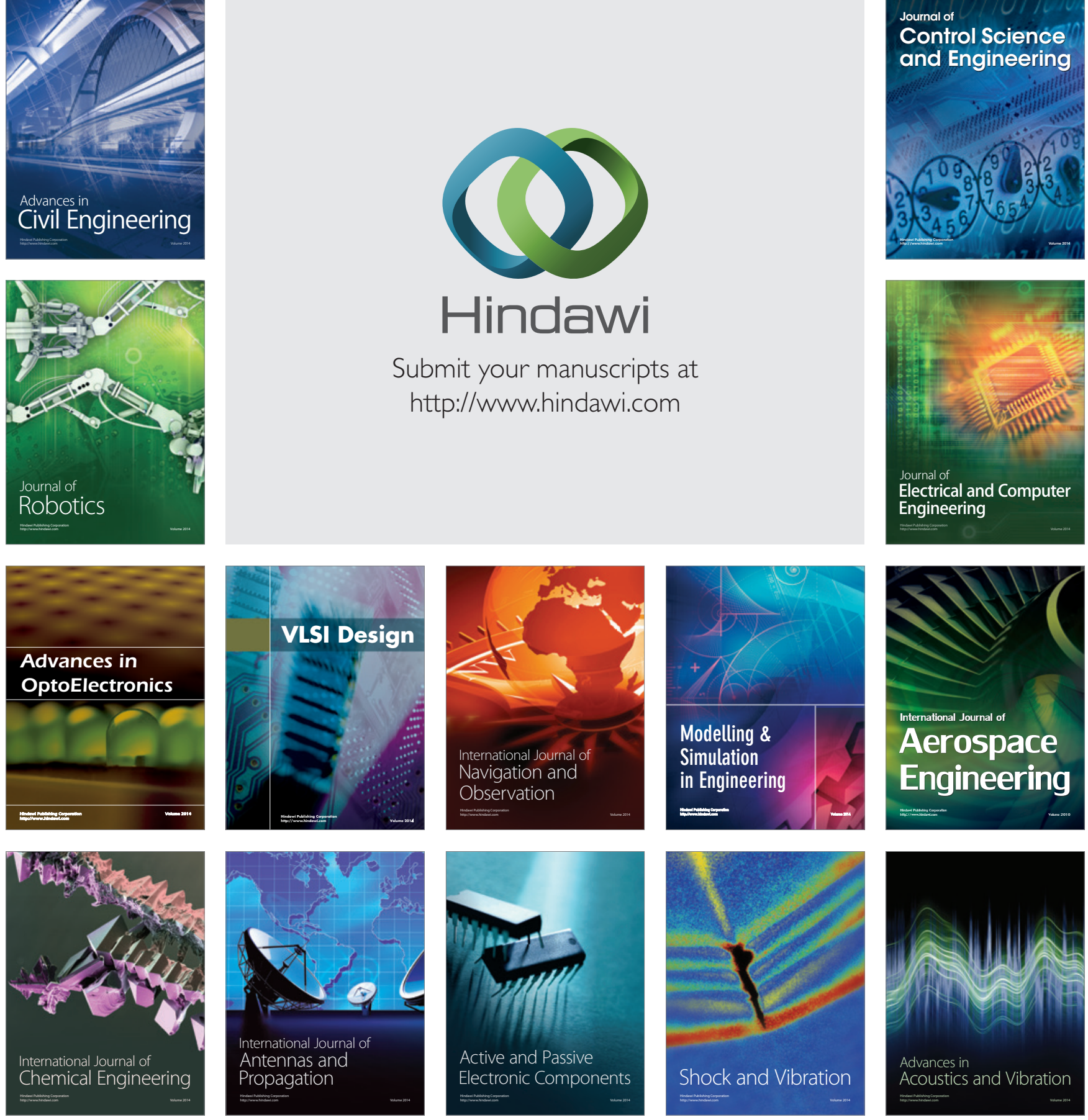